\title{
ORIGINAL ARTICLE \\ Fatigue in persons who have lived with spinal cord injury for $>20$ years
}

\author{
IB Lidal ${ }^{1,6}$, AE Jensen ${ }^{1}$, TW Larsen ${ }^{2,3,4}$ and JK Stanghelle ${ }^{5}$
}

Objectives: This study aims to assess the prevalence of fatigue among persons who have lived with traumatic spinal cord injury (SCI) for $>20$ years, and to compare the results with fatigue scores found among the general population (GP). Another objective was to study the association between fatigue and clinical variables, including mental health, among the study population.

Design: A cross-sectional study.

Setting: Sunnaas Rehabilitation Hospital (SunRH), Norway.

Materials and methods: All SCI survivors $(n=237)$ admitted for rehabilitation at SunRH between 1961 and 1982 were asked to participate. Fatigue was measured with the Fatigue Questionnaire (FQ). Mental health was assessed with the Hospital Anxiety and Depression Scale (HADS). Linear regressions were used to examine those variables with the potential to contribute to fatigue.

Results: A total of 153 persons responded to the $F Q$, and in 39 cases the scores were consistent with fatigue. Surprisingly, the prevalence of fatigue (total fatigue (TF)) did not differ between the study population and the norm. However, the results indicated statistically significantly higher score of physical fatigue (PF) and statistically significantly lower score of mental fatigue among the $\mathrm{SCl}$ group when compared with the GP. Higher fatigue scores were associated with fatigue-causing pharmaceuticals and with higher scores on the HADS-depression subscale.

Conclusions: The prevalence of fatigue was $25 \%$ among persons who had lived with SCl for $>20$ years, and similar to that in the GP. Our results point to medications and mental health aspects as possible contributors to PF severity in $\mathrm{SCl}$.

Spinal Cord (2013) 51, 103-108; doi:10.1038/sc.2012.110; published online 16 October 2012

Keywords: spinal cord injury; fatigue; fatigue questionnaire; mental health

\section{INTRODUCTION}

Fatigue is defined as an overwhelming sense of tiredness, a lack of energy and a frequent feeling of exhaustion. ${ }^{1}$ Commonly distinguished according to mental and physical forms, fatigue is oftentimes a disabling symptom found in a variety of medical and neurologic disorders. ${ }^{2,3}$

Recent decades has seen life-expectancy improvements for persons who sustain a traumatic spinal cord injury (SCI). However, long-term survivors face complex, extraordinary and lifelong challenges. ${ }^{4,5}$ Today, clinicians and researchers find that fatigue is a consequence that magnifies the physical challenges of SCI and diminishes the quality of life. ${ }^{5-7}$ Fawkes-Kirby et al. ${ }^{8}$ found that fatigue was common among individuals with SCI who were seeking outpatient rehabilitation.

In quantitative studies, variables associated with fatigue include injury completeness, pain, spasticity, depression, use of fatiguecausing medications and the use of mobility aids. ${ }^{8-11}$ It is known that depression is common after SCI, and previous reports have documented a relationship between depression and fatigue. ${ }^{2,9}$ There is however a challenge to determine whether depression is a contributor to fatigue or vice versa. ${ }^{2,9}$
In a qualitative study by Hammell et al., ${ }^{12}$ focus group interviews elucidated pain, depression and hopelessness, side effects of medications, poor quality of sleep, spasticity, poor posture, diet, and the effort required to accomplish routine and self-care tasks to be associated with fatigue.

Our clinical experiences found some chronic SCI patients complaining that fatigue symptoms, such as marked lack of energy and feeling exhausted, influenced their daily activities. The study objectives were to assess the prevalence of fatigue among a sample of Norwegians who have had SCI for $>20$ years, and to compare the results with fatigue scores measured among the general Norwegian population. We also wanted to explore the associations between fatigue and the following clinical variables: demographics, lesion characteristics, smoking status, medication, self-perceived health problems and mental health scores. Our main hypotheses are as follows: compared with norms, a higher prevalence of fatigue and higher levels of physical and mental fatigue scores exist among persons with long-term SCI. Our second hypothesis is that demographics, lesion characteristics, smoking status, medication, selfperceived health problems and mental health may influence on fatigue levels in persons with long-term SCI.

\footnotetext{
${ }^{1}$ Sunnaas Rehabilitation Hospital, Nesoddtangen, Norway; ${ }^{2}$ Biostatistics and Epidemiology Unit, Oslo University Hospital, Oslo, Norway; ${ }^{3}$ Centre for Child and Adolescent Mental Health, Eastern and Southern Norway, Oslo, Norway; ${ }^{4}$ Norwegian Centre for Violence and Traumatic Stress Studies, Oslo, Norway and ${ }^{5}$ Sunnaas Rehabilitation Hospital, Faculty of Medicine, University of Oslo, Nesoddtangen, Norway

${ }^{6}$ Current address: Norwegian Knowledge Centre for the Health Services, Nasjonalt kunnskapssenter for helsetjenesten, PO Box 7004 St Olavs plass, N-0130 Oslo, Norway. Correspondence: IB Lidal, Oevre movei 49, Nesoddtangen 1450, Norway.

E-mail: hlidal@broadpark.no and ibl@nokc.no

Received 23 June 2011; revised 17 August 2012; accepted 17 August 2012; published online 16 October 2012
} 


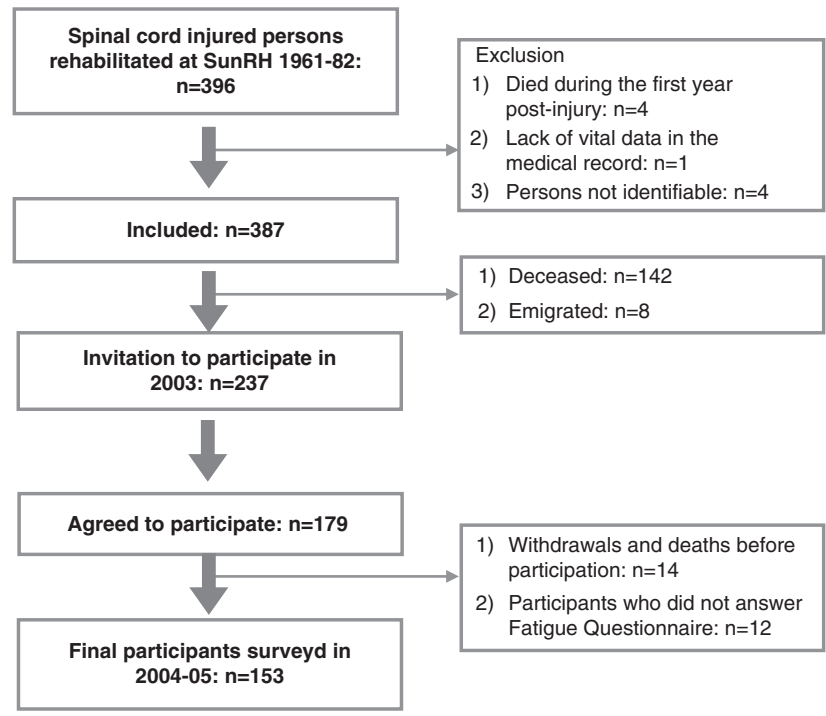

Figure 1 Overview of the inclusion process in the present study.

\section{MATERIALS AND METHODS}

Data for this study were collected as part of a multidimensional cross-sectional survey in 2004-2005,13 (Figure 1). The inclusion criteria were: persons with traumatic SCI who were admitted for initial rehabilitation at Sunnaas Rehabilitation Hospital (SunRH) between 1961 and 1982. The SunRH, affiliated with the Oslo University, is the central spinal unit in Norway with a catchment area of about 2.75 million people (56\% of the Norwegian population). Furthermore, two other spinal units in Norway are located in the Western Coast and in Northern Norway, respectively.

Data on fatigue among the general Norwegian population $(n=2287)$ were collected from a study published by Loge et al. ${ }^{14}$

\section{Participants}

The participants were recruited in 2003 by postal mail; letters were sent to all living persons who received a traumatic SCI between 1961 and 1982 (Figure 1). Collecting data (2004-2005) from the participants took place as follows: (1) a set of self-administered questionnaires was mailed with a stamped return envelope and an appointment for a personal interview; (2) a face-to-face interview and a clinical examination were undertaken. Four participants were interviewed by telephone due to a long distance. Another 21 participants were interviewed and examined in their respective homes. All interviews and examinations were conducted by the same person.

The Norwegian rules on privacy protection demanded consent from respondents and non-respondents before using their data in our research. To gain some information on non-respondents $(n=58)$, a brief questionnaire was mailed to them as well.

The study was approved by The Regional Ethics Committee in Oslo, Norway.

\footnotetext{
Measures

The Fatigue Questionnaire (FQ) was used to assess fatigue. ${ }^{15}$ The FQ was originally developed as a hospital study for chronic fatigue syndrome, to assess symptom severity, the detection of fatigue cases in epidemiological studies and as a valid estimator of change. The FQ has shown acceptable psychometric quality and has been translated into Norwegian. ${ }^{14}$ The FQ asks for fatigue symptoms experienced during the last month compared with how subjects felt when last feeling well. Of the eleven FQ items, seven items assess physical fatigue (PF) and four items assess mental fatigue (MF). All items have four response categories scored on a Likert scale: $0=$ less than usual, $1=$ no more than usual, $2=$ worse than usual, $3=$ much worse than usual; maximum total fatigue $(\mathrm{TF})$ score $=33$. The FQ contains two additional items on duration and extent of fatigue.
}

Loge et al. ${ }^{14}$ suggested the scores to be dichotomised: $0=$ less than usual and no more than usual, and $1=$ worse than usual and much worse than usual; maximum TF dichotomised score $=11$. As in their validation study, we also defined fatigue to exist in cases where the sum of the dichotomised TF reached four or higher. Fatigue 'caseness' was classified if the dichotomised TF sum reached four or higher and fatigue lasted 6 months or longer. ${ }^{14}$

The Hospital Anxiety and Depression Scale (HADS) is often used to assess anxiety and depression. ${ }^{16-19}$ It is based on 14 items, each related to subscales designed to capture both anxiety (HADS-A, seven items) and depression (HADS-D, seven items). All items have four response categories $(0=$ not at all, to $3=$ very often indeed). A score above eight in either of the subscales indicates anxiety or depression, respectively. The psychometric properties are considered to be quite good, and the HADS has been validated in the SCI population. ${ }^{19}$ A Norwegian study has supported the HADS as an instrument with good psychometric properties (factor structure, inter-correlation, homogeneity and internal consistency). ${ }^{20}$ The scale does not include somatic items that could be caused by the SCI itself.

Demographic information on sex, age at injury, age at the time of the study and marital status were collected. Marital status was dichotomised into 'married/ cohabitant' versus 'single'. Employment status was based on a checklist and categorised according to 'employed' (part-time or full-time paid work) and 'not employed' (students, unemployed and homemakers). Smoking habits were categorised as currently 'non-smokers' or 'smokers'. All participants were asked to indicate their present main health problem (open-ended question). ${ }^{4}$ We merged health problems into six categories reflecting the most frequent answers. As the number of variables used in the linear regression model is limited by the size of the studied population, we merged health problems further into three categories. The participants were asked for details about their daily medication, and their responses were merged into the following groups: (1) no daily medication at all; (2) medication that never or seldom causes fatigue as a side effect; (3) medication that often or always causes fatigue as a side effect; (4) medication with two or more drugs that often or always causes fatigue as a side effect. Medication side effects were identified by referencing the Norwegian formulary on pharmaceuticals ('Felleskatalogen') and then classified as either 'never' or 'seldom' causing fatigue or 'often' or 'always' causing fatigue in cases where reported side effects included fatigue, somnolence, drowsiness, weakness, sedation or asthenia.

After a clinical examination, the participants were grouped into 'tetraplegia' or 'paraplegia' according to the level of the SCI. For a neurological classification of SCI we used the ASIA Impairment Scale. ASIA Impairment Scale classes were merged into two groups: ASIA Impairment Scale A-C for 'functionally complete' and D-E for 'functionally incomplete' SCI. Injury aetiology was grouped according to the International Spinal Cord Injury Core Data Set.

\section{Statistical analysis}

Descriptive statistics included proportions, means and s.d., together with simple tests including $\chi^{2}$-tests of independence to compare categorical variables and $t$-tests and Mann-Whitney $U$-tests to compare continuous variables. Cronbach's alphas were created to assess the internal consistencies of fatigue scores. Prevalence of TF was reported with 95\% confidence intervals computed via the Blaker procedure, ${ }^{21}$ and compared to norms ${ }^{14}$ using an exact $\chi^{2}$-test, assuming that the $22 \%$ prevalence reported by Loge et al. was 503 of 2287 persons. Fatigue scores in the SCI population were compared with expected scores based on age and sex in the general population (GP), ${ }^{14}$ using paired sample $t$-tests. Expected scores are mean values in a GP norm sample for the same sex and age group as each person in the study sample. No gold standard exists regarding the clinically significant numerical changes on the FQ. As done by Hjermstad et al., we estimated the differences between the SCI population and the GP by calculating effect size; that is, the mean score difference across groups divided by the s.d. of the norm value. ${ }^{22}$ We regarded effect size of $0.2-0.5$ as small, $0.5-0.8$ as moderate and $>0.8$ as large. ${ }^{23}$

A linear regression was used to study factors that might affect fatigue (TF sum), that is, age, sex, marital status, time since injury, level of injury, ASIA Impairment Scale classification, smoking status, medication use, health problems and mental health as measured with the HADS. ${ }^{24}$ These variables were selected based on judgement and previous research. ${ }^{8-10}$ Collinearity problems were indicated if the variance inflation factor for any covariate exceeded five, and by Spearman correlations. $P$-values $<0.05$ were considered 
significant. Adjustments for multiple testing via the Holm procedure were done within all simple analyses, within norm comparisons and within each regression. Analyses were performed in SPSS (versions 15.0 and 16.0), except the Blaker confidence intervals and Holm adjustment for multiple testing that used R (The R Foundation for Statistical Computing, Vienna, Austria), package Blaker CI for Blaker confidence intervals.

\section{RESULTS}

\section{Population}

A total of 179 individuals (76\%) with traumatic SCI agreed to participate in the study. Information on the non-respondents $(n=58)$ was reported in our paper on health-related quality of life. ${ }^{4}$
At the time of the study, five persons were inaccessible or could not be reached, five persons could not find time to participate, three persons died before data collection, and one person was excluded owing to incomplete data. Of the 165 remaining persons who attended the study, 12 participants (two women; ten men) did not answer the questionnaire portions dealing with fatigue. Thus, data from 153 persons (response $=64.5 \%$ ) were available for the current analyses (Figure 1).

Table 1 shows the characteristics of the study participants, and the differences between persons who scored normal versus persons having a dichotomised sum score of $\geqslant 4$ with the FQ. A total of 57 participants reported daily use of one or more medications with

Table 1 Characteristics of all participants $(n=153)$, including persons without $(n=114)$ and with $(n=39)$ substantial fatigue as measured with the Fatigue Questionnaire, dichotomised scores

\begin{tabular}{|c|c|c|c|c|}
\hline Category & $\begin{array}{l}\text { Total } \\
\mathrm{n}=153\end{array}$ & $\begin{array}{l}F Q<4 \\
\mathrm{n}=114\end{array}$ & $\begin{array}{l}F Q \geqslant 4 \\
n=39\end{array}$ & P-value* \\
\hline \multicolumn{5}{|l|}{ Sex } \\
\hline Male n (\%) & $125(82 \%)$ & $97(85 \%)$ & $28(72 \%)$ & 0.064 \\
\hline Female n (\%) & $28(18 \%)$ & $17(15 \%)$ & $11(28 \%)$ & \\
\hline Mean age at injury (s.d.) & $23.3(9.6)$ & $23.3(10.1)$ & $23.4(8.0)$ & 0.960 \\
\hline Range & $3-55$ & $3-55$ & $8-46$ & \\
\hline Mean age at follow-up (s.d.) & $50.4(9.5)$ & $50.6(9.6)$ & $49.9(9.5)$ & 0.706 \\
\hline Range & $24-81$ & $24-81$ & $34-78$ & \\
\hline Mean time since injury (s.d.) & $27.1(4.2)$ & $27.1(4.4)$ & $26.8(3.7)$ & 0.66 \\
\hline Range & 20-37 & 20-37 & $21-35$ & \\
\hline Employed at follow-up n (\%) & $57(37 \%)$ & $42(37 \%)$ & $15(38 \%)$ & 0.857 \\
\hline Married or cohabitant at follow-up $n(\%)$ & $89(58 \%)$ & $69(61 \%)$ & $20(51 \%)$ & 0.312 \\
\hline Cause of injury & & & & 0.810 \\
\hline Sports $n(\%)$ & $24(16 \%)$ & $18(16 \%)$ & $6(15 \%)$ & \\
\hline Assaults $n(\%)$ & $5(3 \%)$ & $3(3 \%)$ & $2(5 \%)$ & \\
\hline Transport $n(\%)$ & $86(56 \%)$ & $63(55 \%)$ & $23(59 \%)$ & \\
\hline Fall $n(\%)$ & $28(18 \%)$ & $23(20 \%)$ & $5(13 \%)$ & \\
\hline Others or unknown $n(\%)$ & $10(7 \%)$ & $7(6 \%)$ & $3(8 \%)$ & \\
\hline Level of injury & & & & 0.599 \\
\hline Tetraplegia $n(\%)$ & $52(34 \%)$ & $40(35 \%)$ & $12(31 \%)$ & \\
\hline Paraplegia $n(\%)$ & $100(66 \%)$ & $73(64 \%)$ & $27(69 \%)$ & \\
\hline Missing & $n=1$ & $n=1$ & & \\
\hline AIS classification & & & & 0.128 \\
\hline AIS A $n(\%)$ & $106(71 \%)$ & $81(71 \%)$ & $25(64 \%)$ & \\
\hline AIS B $n(\%)$ & $10(7 \%)$ & $9(8 \%)$ & $1(2 \%)$ & \\
\hline AIS C $n(\%)$ & $11(7 \%)$ & $8(7 \%)$ & $3(8 \%)$ & \\
\hline AIS D $n(\%)$ & $18(12 \%)$ & $9(8 \%)$ & $9(23 \%)$ & \\
\hline AIS E $n(\%)$ & $5(3 \%)$ & $4(3 \%)$ & $1(2 \%)$ & \\
\hline Missing & $n=3$ & $n=3$ & & \\
\hline Medication usage & & & & $0.215^{* *}$ \\
\hline No medicine usage $n(\%)$ & $54(35 \%)$ & $43(38 \%)$ & $11(28 \%)$ & \\
\hline Medicine without known fatigue side effect $n(\%)$ & $42(27 \%)$ & $35(31 \%)$ & $7(18 \%)$ & \\
\hline One medicine with fatigue side effect $n(\%)$ & $38(25 \%)$ & $27(24 \%)$ & $11(28 \%)$ & \\
\hline More medicines with fatigue side effect $n(\%)$ & $19(12 \%)$ & $9(8 \%)$ & $10(26 \%)$ & \\
\hline Smoking at follow-up $n(\%)$ & $46(31 \%)$ & $29(26 \%)$ & $17(44 \%)$ & $0.461^{* *}$ \\
\hline Missing & $n=3$ & $n=3$ & & \\
\hline Time period of onset of injury: $1961-1975 n(\%)$ & $76(49 \%)$ & $59(52 \%)$ & $17(44 \%)$ & 0.379 \\
\hline $1976-1982 n(\%)$ & $77(51 \%)$ & $55(48 \%)$ & $22(56 \%)$ & \\
\hline HADS-A (s.d.) & $3.6(3.2)$ & $3.2(2.9)$ & $4.7(3.6)$ & $0.169^{* *}$ \\
\hline Range & $0-13$ & $0-12$ & $0-13$ & \\
\hline HADS-D (s.d.) & $2.2(2.6)$ & $1.6(1.9)$ & $3.8(3.5)$ & $0.008^{* *}$ \\
\hline Range & $0-13$ & 0-9 & 0-13 & \\
\hline
\end{tabular}

Abbreviations: AIS, ASIA Impairment Scale (ASIA, American Spinal Injury Association International Neurological Standards for Classification of Spinal Cord Injury); FQ, Fatigue Questionnaire; HADS-A, Hospital Anxiety and Depression Scale, anxiety; HADS-D, depression; s.d., standard deviation.

${ }^{*} P$-value: difference between $\mathrm{FQ}<4$ and $\mathrm{FQ} \geqslant 4$.

${ }^{*} P$-value: adjusted $P$-value for multiple testing via the Holm procedure (unadjusted $P$-value $<0.05$ ). 
fatigue as a known side effect, whereof medications for cardiovascular disease, predominantly antihypertensiva, analgesics and/or medications for spasticity were most common. Twenty-two per cent of the participants reported 'no health-problems', 16\% reported 'severe pain problems', $13 \%$ reported 'problems related to the urinary tract', $10 \%$ reported 'musculoskeletal problems' and another $10 \%$ assessed 'my disability' as their main health problem. 'Other health problem areas' were reported with proportions $<10 \%$, including pressure sores, spasticity, and bowel related difficulties. Mean HADS total score was 5.8 (s.d., 5.02), mean HAD-A was 3.6 (s.d., 3.2), mean HAD-D was 2.2 (s.d., 2.6).

\section{Fatigue}

The internal consistencies for the TF, PF and MF segments were 0.81 , 0.81 and 0.68 , respectively. No collinearity problems were shown; the highest variance inflation factor was 1.7 both for TF, PF and MF. Also, the highest Spearman correlation (in absolute value) between covariates in our models was -0.56 between the two health problem dummies; all other Spearman correlations were 0.35 or lower in absolute value.

A total of 39 persons (25\%; 95\% confidence interval: $19-33 \%)$ of the SCI-participants reported a dichotomised TF sum equal to or above four, indicating fatigue. The prevalence of fatigue was not statistically different from the GP (dichotomised TF sum of 22\%, 95\% CI $20 \%-24 \%), P=0.316$. In this study, no incidents of 'caseness' were found.

Table 2 shows the results for TF, PF and MF compared to the GP. ${ }^{14}$ The ESs of the differences were small overall (TF: 0.11, PF: 0.21, and MF: 0.14), indicating small clinical differences.

Table 3 contains results from the linear regressions for TF, PF and MF, respectively. For TF and PF, the use of more medications with fatigue as a possible side effect and higher scores with the HADS depression subscale are associated with higher fatigue scores. None of the covariates investigated were related to MF, consistent with an adjusted $R^{2}$ about 0 . For the TF and PF scores the models had some explanatory power, although the adjusted $R^{2}$ was rather low for these models also.

\section{DISCUSSION}

The most interesting finding from the present investigation is that the prevalence of fatigue in individuals who incurred a SCI $>20$ years ago does not differ from the prevalence found among the GP. However, some differences were seen with the subscales, as the SCI population scored statistically significantly higher with the PF and statistically significantly lower with the MF dimension of the FQ compared with norms. The daily use of more medications with a documented risk of fatigue as a side effect, and also higher scores on the HADS depression subscale, were associated with higher PF scores in persons with longstanding SCI.
We hypothesised a higher prevalence of fatigue and higher levels of physical and mental fatigue among persons with SCI of $>20$ years duration compared with the norm, but the findings did not support our theory. One explanation may be that persons with higher levels of fatigue may be less likely to participate in surveys. The study's results may have been influenced by a bias among participants; however, the response rate of $76 \%$ is relatively high. A point of concern is that the FQ norm data were collected about 8 years previous to our data, and we are not aware of more recent FQ norm data from Norway. The norm fatigue scores may have risen somewhat throughout the years, as more people are surviving longer with chronic and serious diseases. Thus, the prevalence of fatigue in the study population may be even less different from the norms. The findings also indicate that fatigue is less prevalent among our participants compared with other studies on SCI (7-10). The variability of findings between studies may be related to differences in study populations (age, time since injury, injury characteristics, comorbidity and so on) and methodological disparities, including fatigue measures used.

Despite a higher PF level and lower MF level in our SCI group compared with the norm, the differences probably do not represent any clinical importance (small effect sizes). However, our results suggest that $\mathrm{PF}$ is more prominent than MF components. A number of factors may cause high PF scores in persons with SCI, including usage of $\beta$-blockers, deconditioning and pain. The prevalence of additional conditions such as cardiovascular diseases and demanding more medications increases as people age. Many persons with SCI use the same medications for several years. According to our results, the daily intake of a number of antihypertensives, analgesics and medications for spasticity, probably affects PF, but to a small degree the mental dimensions of fatigue in persons with longstanding SCI. The findings support Lee et al. ${ }^{10}$ who also found that usage of a number of analgesics was statistically significantly contributing to higher fatigue scores as measured with the Fatigue Severity Scale. However, the Fatigue Severity Scale (unidimensional scale) does not distinguish mental and physical dimensions of fatigue.

The study participants scored better with the MF compared with norms. This could be due to a responder bias, as discussed above, or to a survival effect (see below). Another explanation could be that the FQ has not good enough discriminant validity for use in a population with longstanding SCI. The FQ has not been validated for use in this population. The effect sizes of the differences in fatigue scores were however small, thus indicating an insignificant clinical difference.

In our previous study on health-related quality of life, ${ }^{4}$ the same SCI-sample scored a non-significant difference with the mental health domain of the SF-36 compared with normal parameters, and overall better health-related quality of life compared with other SCIpopulations. We suggested that one explanation might be that individuals with SCI often develop adaptive strategies over time that allow them to cope with complications and deterioration in

Table 2 Fatigue Questionnaire, differences between mean expected scores $(n=2287)$ and mean scores in the study SCI-population $(n=153)$

\begin{tabular}{|c|c|c|c|c|c|}
\hline & SCl mean (s.d.) & Expected mean (s.d.) ${ }^{a}$ & Mean difference (s.d.) & $95 \% \mathrm{Cl}$ & P-value* \\
\hline Physical fatigue & $8.5(2.6)$ & $7.8(0.4)$ & $-0.65(2.66)$ & $-1.07--0.22$ & $0.003(0.009)$ \\
\hline Mental fatigue & $4.2(1.0)$ & $4.4(0.1)$ & 0.19 (1.04) & $0.03-0.36$ & $0.024(0.048)$ \\
\hline Total fatigue & $12.6(3.1)$ & $12.2(0.5)$ & $-0.44(3.18)$ & $-0.95-0.07$ & 0.091 \\
\hline
\end{tabular}

Abbreviations: $\mathrm{Cl}$, confidence interval; s.d., standard deviation.

aDerived from Loge et al. ${ }^{14}$

${ }^{*} P$-values corrected for multiple testing (Holm correction) in parentheses for significant $P$-values. 
Table 3 Multiple linear regression results: TF, PF and $\mathrm{MF}^{*}$ in the $\mathrm{SCl}$ sample $(n=153)$ measured with the Fatigue Questionnaire and selected factors possibly affecting total fatigue

\begin{tabular}{|c|c|c|c|c|c|c|c|c|c|}
\hline \multirow[t]{2}{*}{ Variables } & \multicolumn{3}{|c|}{ Total fatigue } & \multicolumn{3}{|c|}{ Physical fatigue } & \multicolumn{3}{|c|}{ Mental fatigue } \\
\hline & Coefficient & $95 \% \mathrm{Cl}$ & $\mathrm{P}$-value & Coefficient & $95 \% \mathrm{Cl}$ & P-value & Coefficient & $95 \% \mathrm{Cl}$ & P-value \\
\hline \multicolumn{10}{|l|}{ Sex } \\
\hline $\begin{array}{l}\text { Female } \\
\text { Male (ref.) }\end{array}$ & -0.108 & $1.423-1.206$ & 0.871 & -0.178 & $-1.256-0.900$ & 0.744 & 0.091 & $-0.385-0.566$ & 0.707 \\
\hline \multicolumn{10}{|l|}{ Marital status } \\
\hline $\begin{array}{l}\text { Married/cohabitant } \\
\text { Other (ref.) }\end{array}$ & 0.739 & $-0.289-1.768$ & 0.157 & 0.638 & $-0.203-1.478$ & 0.136 & 0.078 & $-0.294-0.450$ & 0.681 \\
\hline Age at interview (2004/05) & -0.020 & $-0.078-0.039$ & 0.507 & -0.015 & $-0.063-0.033$ & 0.533 & -0.003 & $-0.024-0.018$ & 0.762 \\
\hline Time since injury & 0.064 & $-0.055-0.182$ & 0.290 & 0.053 & $-0.044-0.151$ & 0.283 & 0.011 & $-0.032-0.054$ & 0.601 \\
\hline \multicolumn{10}{|l|}{ Level of SCl } \\
\hline $\begin{array}{l}\text { Tetraplegia } \\
\text { paraplegia (ref.) }\end{array}$ & 0.028 & $-1.027-1.082$ & 0.959 & 0.281 & $-0.581-1.143$ & 0.520 & -0.221 & $-0.603-0.160$ & 0.253 \\
\hline \multicolumn{10}{|l|}{ AIS } \\
\hline $\begin{array}{l}\text { A-C } \\
\text { D-E (ref.) }\end{array}$ & -0.817 & $-2.203-0.569$ & 0.246 & -0.681 & $-1.797-0.434$ & 0.229 & -0.207 & $-0.709-0.295$ & 0.416 \\
\hline \multicolumn{10}{|l|}{ Smoking status } \\
\hline $\begin{array}{l}\text { Smoking } \\
\text { Non-smoking (ref.) }\end{array}$ & -0.376 & $-1.530-0.778$ & 0.521 & -0.337 & $-1.283-0.610$ & 0.483 & -0.046 & $-0.464-0.371$ & 0.827 \\
\hline \multicolumn{10}{|l|}{ Medication usage } \\
\hline $\begin{array}{l}\text { Medicine without known fatigue } \\
\text { side effect }\end{array}$ & 0.489 & $-0.795-1.773$ & 0.452 & 0.367 & $-0.677-1.410$ & 0.488 & 0.065 & $-0.400-0.529$ & 0.783 \\
\hline $\begin{array}{l}\text { One medicine with fatigue side } \\
\text { effect }\end{array}$ & 0.490 & $-0.795-1.774$ & 0.452 & 0.288 & $-0.759-1.334$ & 0.588 & 0.149 & $-0.315-0.614$ & 0.526 \\
\hline $\begin{array}{l}\text { More medicines with fatigue } \\
\text { side effect } \\
\text { No medicine (ref.) }\end{array}$ & 2.019 & $0.268-3.770$ & $0.024^{*}$ & 1.925 & $0.495-3.355$ & $0.009^{\dagger}$ & 0.048 & $-0.586-0.681$ & 0.882 \\
\hline \multicolumn{10}{|l|}{ Main health problemb } \\
\hline No health problem & -0.070 & $-1.319-1.179$ & 0.911 & -0.178 & $-1.201-0.846$ & 0.732 & 0.072 & $-0.380-0.524$ & 0.753 \\
\hline Pain & 0.458 & $-1.014-1.930$ & 0.539 & 0.042 & $-1.166-1.250$ & 0.945 & 0.405 & $-0.127-0.938$ & 0.135 \\
\hline Other (ref.) & & & & & & & & & \\
\hline \multicolumn{10}{|l|}{ Mental health } \\
\hline HADS-A & 0.074 & $-0.122-0.270$ & 0.458 & 0.043 & $-0.118-0.204$ & 0.596 & 0.033 & $-0.380-0.104$ & 0.364 \\
\hline HADS-D & 0.377 & $0.142-0.612$ & $0.002^{* *}$ & 0.358 & $0.165-0.551$ & $<0.001^{\ddagger}$ & 0.014 & $-0.071-0.100$ & 0.738 \\
\hline Adjusted $R^{2}$ & 0.136 & $F_{14,129}=2.604$ & $=0.002$ & 0.164 & $F_{14,130}=3.017$ & $<0.001$ & -0.035 & $F_{14,129}=2.604$ & 0.811 \\
\hline
\end{tabular}

Abbreviations: AIS, ASIA Impairment Scale (ASIA, American Spinal Injury Association International Neurological Standards for Classification of Spinal Cord Injury); CI, confidence interval; ref., reference category; HADS-A, Hospital Anxiety and Depression Scale-anxiety segments; HADS-D,_- depression segments.

ref., reference category; HADS-A, Hospital Anxiety and Depression Scate
aTF, PF and MF summary score (Likert) was the dependent variable.

'The main health problem categories: 'problems related to the urinary tract'; 'musculoskeletal problems', 'my disability' and 'other health problem areas'; were for analytical purpose merged into one category, namely 'Other'. ${ }^{*} P=0.314$ after Holm correction for multiple testing; ${ }^{*} P=0.026$ after Holm correction for multiple testing; ${ }^{\dagger} P=0.113$ after Holm correction for multiple testing; $\ddagger P=0.005$ after Holm correction for multiple testing.

function. The present MF results (that is, better MF outcomes compared with norms and no association between MF and the HADS) might reflect the same strategies, and also an effect of healthier persons/psychological stability analogous to survival effects. Instead, our results point to a relationship between PF and the HADS depression subscale.

We could not prove any association between fatigue and demographic and clinical variables such as sex, age, time since injury, marital status, level and extent of SCI, health problems or smoking. In previous studies, some of the above mentioned variables have shown relationships to fatigue. ${ }^{8-10}$ As previously mentioned, the variability of findings among studies may be related to methodological differences, and need further clarification.

\section{LIMITATIONS}

Information on the selection of patients for rehabilitation at SunRH in the 1960s and the early 1970s is lacking, and whether the studied population accurately represents the Norwegian SCI population is therefore debatable. However, based on two studies and an estimated 1 -year mortality rate of $20 \%,{ }^{26,27}$ we calculated that our cohort ( $n=387$ ) includes $\sim 50 \%$ of all Norwegians injured between 1961 and 1982 who survived at least 1 year after SCI (calculations available on request).

The FQ is neither validated in a SCI population, nor has it been used in previous SCI-studies, thus making comparisons of our findings with other studies on SCI and fatigue restricted. It has been claimed that the presence of primary physical or cognitive 
dysfunction may confound interpretations of the responses. ${ }^{28}$ Also, Cronbach's alpha was slightly below 0.70 for the MF scale.

The relationship between depression and fatigue is complicated, and from this study we do not know whether depression brings PF or vice versa. The cross-sectional design does not capture changes over time, nor is it possible to draw causal connections.

In conclusion, this study indicates that the prevalence of fatigue in longstanding SCI does not exceed the prevalence of fatigue in the GP. PF was prevalent in $25 \%$ of those who incurred a traumatic SCI $>20$ years ago. Higher TF and PF scores seem to be associated with higher scores on the HADS depression subscale and also with the number of daily medications. With aging, it is important to consider drug side effects and tolerability when dealing with fatigue-like symptoms.

This study does not make inferences about causality, and it was not aiming to establish an understanding of how to treat fatigue. Randomised controlled trials or controlled before-and-after studies are needed before causal statements and effective treatment suggestions can be made.

\section{DATA ARCHIVING}

There were no data to deposit.

\section{CONFLICT OF INTEREST}

The authors declare no conflict of interest.

\section{ACKNOWLEDGEMENTS}

First and foremost we want to thank the study participants, the Norwegian Association of the Disabled and the Norwegian Association for Spinal Injuries. We also want to thank those who contributed financial support to the project: the Norwegian Foundation for Health and Rehabilitation (EXTRA), the Sunnaas Rehabilitation Hospital and the Eastern Norway Health Authority (Helse Øst). Thanks to Vegard Strøm for his valuable comments.

1 Herlofson K, Larsen JP. Measuring fatigue in patients with Parkinson's disease-The Fatigue Severity Scale. Eur J Neurol 2002; 9: 595-600.

2 Krupp LB, LaRocca NG, Muri-Nash J, Steinberg AD. The Fatigue Severity Scale: application to patients with multiple sclerosis and systemic lupus erythematosus. Arch Neurol 1989; 46: 1121-1123.

3 Chaudhuri A, Behan PO. Fatigue in neurological disorders. The Lancet 2004; 363 978-988.

4 Lidal IB, Veenstra M, Hjeltnes N, Biering-Sørensen F. Health-related quality of life in persons with long-standing spinal cord injury. Spinal Cord 2008; 46: 710-715.
5 McColl MA, Charlifue S, Glass C, Lawson N, Savic G. Aging gender, and spinal cord injury. Arch Phys Med Rehabil 2004; 85: 363-367.

6 Levi R, Hultling C, Nash MS, Seiger A. The Stockholm Spinal Cord Injury Study: 1. Medical problems in a regional SCl population. Paraplegia 1995; 33: 308-315.

7 Wijesuriya N, Tran Y, Middleton J, Craig A. Impact of fatigue on the health-related quality of life in persons with spinal cord injury. Arch Phys Med Rehabil 2012; 93: 319-324.

8 Fawkes-Kirby TM, Wheeler MA, Anton HA, Miller WC, Townson AF, Weeks CA. Clinical correlates of fatigue in spinal cord injury 2. Spinal Cord 2008; 46: 21-25.

9 Anton HA, Miller WC, Townson AF. Measuring fatigue in persons with spinal cord injury. Arch Phys Med Rehabil 2008; 89: 538-542.

10 Lee AKY, Miller WC, Townson AF, Anton HA. Medication use is associated with fatigue in a sample of community-living individuals who have a spinal cord injury: a chart review. Spinal Cord 2010; 48: 429-433.

11 Saunders LL, Krause JS. Behavioral factors related to fatigue among persons with spinal cord injury. Arch Phys Med Rehabil 2012; 93: 313-318.

12 Hammell KW, Miller WC, Forwell SJ, Forman BE, Jacobsen BA. Fatigue and spinal cord injury: A qualitative analysis. Spinal Cord 2009; 47: 44-49.

13 Lidal IB, Snekkevik H, Aamodt G, Hjeltnes N, Stanghelle JK, Biering-Sørensen F. Mortality after spinal cord injury in Norway. J Rehabil Med 2007; 39: 145-151.

14 Loge JH, Ekeberg 0, Kaasa S. Fatigue in the general Norwegian population: Normative data and associations 86. J Psychosom Res 1998; 45: 53-65.

15 Chalder T, Berelowitzt G, Pawlikowska T, Watts L, Wessely S, Wright D et al. Development of a fatigue scale. J Psychosom Res 1993; 37: 147-153.

16 Zigmond AS, Snaith RP. The Hospital Anxiety and Depression Scale. Acta Psychiatr Scand 1983; 67: 361-370.

17 Bjelland I, Dahl AA, Haug TT, Neckelmann D. The validity of the Hospital anxiety and depression scale. An updated literature review. J Psychosom Res 2002; 52: 69-77.

18 Stordal E, Bjartveit Krüger M, Dahl NH, Krüger $\varnothing$, Mykletun A, Dahl AA. Depression in relation to age and gender in the general population: the Nord-Trøndelag Health Study (HUNT). Acta Psychiatr Scand 2001; 104: 210-216.

19 Woolrich RA, Kennedy P, Tasiemski T. A preliminary psychometric evaluation of the Hospital Anxiety and Depression Scale (HADS) in 963 people living with a spinal cord injury. Psycol Health Med 2006; 11: 80-90.

20 Mykletun A, Stordal E, Dahl AA. Hospital anxiety and depression (HAD) scale: Factor structure, item analyses and internal consistency in a large population. BJ Psych 2001; 179: 540-544.

21 Blaker $\mathrm{H}$. Confidence curves and improved exact confidence intervals for discrete distributions. Can J Stat 2000; 28: 783-798.

22 Hjermstad MJ, Fosså SD, Oldervoll L, Holte H, Jacobsen AB, Loge JH. Fatigue in longterm Hodgkin's Disease survivors: A follow-up study. J Clin Oncol 2005; 23: 6587-6595.

23 Cohen J (1988). Statistical Power Analysis for the Behavioral Sciences, 2nd edn, Hillsdale, NJErlbaum.

24 Harrell F, Frank E. Regression Modeling Strategies: with Applications to Linear Models, Logistic Regression, and Survival Analysis. Vol. XXIV, 1st edn (2001), Corr 2nd printing (2001). Springer Series in Statistics, 570pp.

25 Westgren N, Levi R. Quality of life and traumatic spinal cord injury. Arch Phys Med Rehabil 1998; 79: 1433-1439.

26 Gjone R, Nordlie L. Incidence of traumatic paraplegia and tetraplegia in Norway: a statistical survey of the years 1974 and 1975. Paraplegia 1979; 16: 88-93.

27 Hagen EM, Eide GE, Rekand T, Gilhus NE, Gronning MA. A 50-year follow-up of the incidence of traumatic spinal cord injuries in Western Norway. Spinal Cord 2010; 48 313-318.

28 Dittner AJ, Wessely SC, Brown RG. The assessment of fatigue. A practical guide for clinicians and researchers. J Psychosom Res 2004; 56: 157-170. 